\section{(2) OPEN ACCESS}

\title{
New insights in post-traumatic headache with cluster headache phenotype: a cohort study
}

\author{
Lou Grangeon, ${ }^{1,2}$ Emer O'Connor, ${ }^{3}$ Chun-Kong Chan, ${ }^{1}$ Layan Akijian, ${ }^{1}$ \\ Thanh Mai Pham Ngoc, ${ }^{4}$ Manjit Singh Matharu (i) ${ }^{1,5}$
}

${ }^{1}$ Headache and Facial Pain Group, UCL Queen Square Institute of Neurology, London, UK

${ }^{2}$ Department of Neurology, University Hospital Centre Rouen, Rouen, Normandie, France

${ }^{3}$ Department of Molecular Neuroscience, UCL Queen Square Institute of Neurology, London, UK

${ }^{4}$ Mathematics Institute of Orsay, Paris-Sud University, CNRS and Paris-Saclay University, Orsay,

Île-de-France, France

${ }^{5}$ Headache and Facial Pain Group, The National Hospital for Neurology and Neurosurgery, London, UK

Correspondence to Dr Manjit Singh Matharu, Headache and Facial Pain Group, UCL Queen Square Institute of Neurology, London WC1N 3BG, UK; m.matharu@ uclmail.net

Received 29 December 2019 Revised 24 February 2020 Accepted 25 February 2020 Published Online First 7 May 2020

Check for updates

(c) Author(s) (or their employer(s)) 2020. Re-use permitted under CC BY-NC. No commercial re-use. See rights and permissions. Published by BMJ.

To cite: Grangeon L,

O'Connor $\mathrm{E}$, Chan C-K, et al.

J Neurol Neurosurg Psychiatry 2020:91:572-579.

\section{ABSTRACT}

Objectives To define the characteristics of posttraumatic headache with cluster headache phenotype (PTH-CH) and to compare these characteristics with primary $\mathrm{CH}$.

Methods A retrospective study was conducted of patients seen between 2007 and 2017 in a headache centre and diagnosed with PTH-CH that developed within 7 days of head trauma. A control cohort included 553 patients with primary $\mathrm{CH}$ without any history of trauma who attended the headache clinic during the same period. Data including demographics, attack characteristics and response to treatments were recorded.

Results Twenty-six patients with PTH-CH were identified. Multivariate analysis revealed significant associations between PTH-CH and family history of $\mathrm{CH}$ (OR 3.32, 95\% Cl 1.31 to 8.63), chronic form (OR 3.29, $95 \% \mathrm{Cl} 1.70$ to 6.49$)$, parietal (OR $14.82,95 \% \mathrm{Cl} 6.32$ to 37.39 ) or temporal (OR $2.04,95 \% \mathrm{Cl} 1.10$ to 3.84 ) location of pain, and presence of prominent cranial autonomic features during attacks (miosis OR 11.24, 95\% Cl 3.21 to 41.34; eyelid oedema OR $5.79,95 \% \mathrm{Cl}$ 2.57 to 13.82 ; rhinorrhoea $\mathrm{OR} 2.65,95 \% \mathrm{Cl} 1.26$ to 5.86; facial sweating OR 2.53, 95\% Cl 1.33 to 4.93). Patients with PTH-CH were at a higher risk of being intractable to acute (OR $12.34,95 \% \mathrm{Cl} 2.51$ to 64.73 ) and preventive (OR $16.98,95 \%$ Cl 6.88 to 45.52 ) treatments and of suffering from associated chronic migraine (OR 10.35, 95\% Cl 3.96 to 28.82).

Conclusion This largest series of PTH-CH defines it as a unique entity with specific evolutive profile. Patients with PTH-CH are more likely to suffer from the chronic variant, have marked autonomic features, be intractable to treatment and have associated chronic migraine compared with primary $\mathrm{CH}$.

\section{INTRODUCTION}

Post-traumatic headache (PTH) is defined as headache that develops within 7 days of head trauma. ${ }^{1}$ It is difficult to determine the true prevalence of this type of headache; however, it appears to be more frequently associated with mild rather than severe head injury. ${ }^{2}$ Tension-type headache and migraine without aura are the usual associated phenotypes. ${ }^{4}$ Trigeminal autonomic cephalalgias (TAC) have been reported following head injury ${ }^{5-7}$; however, only two cases fulfil the International Classification of Headache Disorders (ICHD) criteria for posttraumatic headache and cluster headache $(\mathrm{CH})$, the vast majority of cases being described with a prolonged or unknown latency between $\mathrm{CH}$ onset and head trauma. ${ }^{8-10}$

Described as one of the most painful conditions known to humans, $\mathrm{CH}$ is characterised by a strictly unilateral headache with associated ipsilateral cranial autonomic features. ${ }^{1}$ It is the most frequent TAC, with an estimated lifetime prevalence of $0.12 \%$. There have been several advances in delineating the pathophysiology of $\mathrm{CH}$ over the last decade. Recent functional imaging data suggest that ipsilateral hypothalamic activation with subsequent trigeminovascular and cranial autonomic activation underpins the pathogenesis of $\mathrm{CH} .{ }^{1112}$ However, its relationship with head trauma remains unclear. According to Lambru and Matharu, ${ }^{8}$ there are three hypotheses explaining the link between head injury and $\mathrm{CH}$. First, $\mathrm{CH}$ may occur as a direct result of head trauma; second, head trauma may only increase the risk of developing $\mathrm{CH}$; and finally that the personality traits associated with $\mathrm{CH}$ may predispose to head trauma.

Although most PTHs resolve within 12 months of injury, approximately 18\%-33\% of PTHs persist, leading to loss of work capacity and significant fiscal consequences. ${ }^{13} 14$ Postconcussion syndrome, with associated depression, cognitive dysfunction and insomnia, also contributes to this economic burden. ${ }^{15}$ This highlights both the clinical and medicolegal imperative to define post-traumatic headache with cluster headache phenotype (PTH$\mathrm{CH}$ ) accurately. Thus, this cohort study attempts to define the characteristics of PTH-CH and compare them with primary $\mathrm{CH}$.

\section{MATERIALS AND METHODS Patients}

This retrospective cohort study was conducted in a tertiary headache centre at the National Hospital for Neurology and Neurosurgery (Queen Square, London, UK) between January 2007 and May 2017. All consecutive patients with prior head trauma and diagnosed with $\mathrm{CH}$ according to the current International Classification of Headache Disorders - Third Edition (ICHD-3) diagnostic criteria were assessed. ${ }^{1}$ All patients were evaluated and examined by a neurologist. After careful review of medical records, we only included patients with a latency period between head trauma and the first $\mathrm{CH}$ attack of 7 days at the most, thus meeting the ICHD criteria for both post-traumatic headache and $\mathrm{CH}$. 
Box 1 Definitions of intractable response to treatment

Adequate trial performed.

- Appropriate dose: decision left to the clinical physician.

- Appropriate length of time: At least 1 month for melatonin trial. At least 3 months for all other preventive therapies.

Failed trial.

- Unsatisfactory response:

Less than $50 \%$ reduction in mean attack frequency for preventive treatment.

Less than $50 \%$ reduction in pain at least $50 \%$ of the time for acute treatment.

- Side effects requiring cessation of treatment.

- Contraindications to use.

Intractable to acute treatment.

- Failure to respond within 15 min of subcutaneous sumatriptan use.

- Failure within $30 \mathrm{~min}$ of high-dose and flow rate oxygen use.

Intractable to preventive treatment.

- Failure of at least four classes among the following:

- Verapamil.

- Lithium.

- Topiramate.

- Gabapentin.

- Methysergide.

- Melatonin

Modified from the European Headache Federation consensus guideline and Goadsby et $a l^{17}$

\section{Data collection}

Demographic characteristics, attack frequency, duration, laterality and associated symptoms including autonomic or migrainous features were recorded. Each site of pain and/or referred pain was categorised as orbital, temporal, frontal, parietal, occipital or cheek. Patients were diagnosed with episodic $\mathrm{CH}(\mathrm{ECH})$ when attacks occurred in clusters lasting weeks to months, with remissions lasting longer than 3 months, and with chronic $\mathrm{CH}(\mathrm{CCH})$ if they had no such attack-free periods. Two independent neurologists established a diagnosis of probable $\mathrm{CH}$ in cases where one of the ICHD-3 criteria was not met. We also recorded any other associated headaches using the ICHD-3 diagnostic criteria. The patients underwent the same assessment of diagnosis and specific characteristics of headache over the years by the same neurologist team. The data were prospectively recorded during clinic visits.

In order to evaluate the response to acute and preventive treatment, we used a modified definition of intractable $\mathrm{CH}$ based on the European Headache Federation and the work of Goadsby et al (box 1). ${ }^{16}{ }^{17} \mathrm{~A}$ satisfactory response to a trial was defined as a $50 \%$ or more reduction in mean attack frequency for preventive medications and as a $50 \%$ or more reduction in pain at least $50 \%$ of the time for acute medications. ${ }^{18}$ Patients were classified as indeterminate in case of insufficient data (less than four trials of preventive treatment or lack of either sumatriptan injection or high-flow oxygen as acute treatment). The response to treatment was determined based on the last available clinical letter.

For each patient, we evaluated the cause and mechanism of head trauma, initial symptoms, such as post-traumatic amnesia or loss of consciousness, and neuroimaging. In case of headache of greater than 3 months' duration, the PTH-CH was called 'persistent'. According to the International Headache Society classification, we defined head trauma as severe/moderate or mild.

A family history of $\mathrm{CH}$ was collected according to proxy reports from patients. To confirm a diagnosis of $\mathrm{CH}$, available affected relatives were interviewed by a neurologist either over the phone or in person at the clinic. Relatives who had died or were unavailable for interview were included if they had previously been diagnosed with $\mathrm{CH}$ by a neurologist or had a history highly suspicious for $\mathrm{CH}$.

\section{Comparison with a control cohort}

In order to ascertain specific characteristics in patients with PTH$\mathrm{CH}$, a comparison with a control cohort was done. All patients who attended the headache clinic during the same period and who fulfilled the criteria for primary $\mathrm{CH}$ were included in our control cohort $(n=631)$. The same evaluation and data collection conducted in the PTH-CH group were performed in the cohort. In addition, any patient with history of head trauma $(\mathrm{n}=52)$ or patients diagnosed with $\mathrm{CH}$ considered as secondary to another disorder $(\mathrm{n}=26)$ were excluded from the final control cohort $(n=553)$, given the possible impact on pain pathways of those events or disorders. No difference between patients with $\mathrm{PTH}-\mathrm{CH}$ and patients with primary $\mathrm{CH}$ was found with regard to demographic data, such as current age, sex, age of onset and follow-up duration. Therefore, this allowed further comparisons regarding clinical characteristics.

\section{Statistics}

Descriptive statistics were expressed as mean with SD. The two groups of patients were compared using a Mann-Whitney non-parametric test for continuous data and a Fisher's exact or $\chi^{2}$ test for categorical data. For the multivariate analysis, we adopted a three-step approach. First, we balanced our two groups of patients with PTH-CH and primary $\mathrm{CH}$. Indeed, when dealing with highly imbalanced classes, classification rules tend to be overwhelmed by the majority class to the detriment of the minority class. A common statistical approach to deal with imbalanced learning is to alter the class distribution to get a more balanced sample. Second, we performed variable selection to select a subset of relevant covariates for our model construction and finally a logistic regression with the previously selected features. Note that we did not split the data into a training and a test set as done usually. Indeed, due to the highly imbalanced data set, adopting the standard holdout strategy would lead to high variance estimates of the accuracy measure. Instead, we adopted a specific holdout version adapted to imbalanced learning. More specifically, we handled missing values by imputation techniques based on random decision forests. Then we used the ROSE (Random Over Sampling Examples) algorithm to balance the data. ${ }^{19}$ The ROSE function creates an artificial balanced sample according to a smoothed bootstrap approach. Then, on the ROSE sample we performed variable selection to get a subset of important covariates to feed into the logistic regression. We resorted to the powerful LASSO (Least Absolute Shrinkage and Selection Operator) algorithm to select relevant explanatory variables. ${ }^{20}$ The LASSO performs automatic variable selection and is capable of selecting groups of correlated variables. Eventually, we performed a logistic regression with the variables selected by the LASSO. All the numerical results were performed with the R software. The randomForest, ROSE and glmnet packages were used. The threshold for statistical significance was set to $\mathrm{p} \leq 0.05$. 


\section{RESULTS}

\section{Demographics and clinical characteristics}

We identified 26 patients diagnosed with PTH-CH within the defined study period, of whom 19 (73.0\%) were male (tables 1 and 2). PTH-CH was persistent in all of them. The mean age was 48.4 years (SD 11.2) and the mean age at $\mathrm{CH}$ onset was 31.8 years old (SD 13.5). The mean follow-up time in our headache clinic was 6.5 years (SD 13.5). Five (19.2\%) patients with PTH-CH were diagnosed with ECH or probable ECH phenotype and $21(80.7 \%)$ with $\mathrm{CCH}$ or probable $\mathrm{CCH}$ phenotype.

Of the 21 patients with $\mathrm{CCH}$ phenotype, 15 were chronic at onset. Of the remaining patients, six developed the ECH phenotype immediately after the injury. The mean duration of attacks was $87.3 \mathrm{~min}$ (SD 55) and the mean frequency was 3.3 daily (SD 1.8). At least one autonomic feature was present during the attacks in the entire case group, with restlessness during an attack reported in all but two patients. At least one migrainous feature was reported in $24(92.3 \%)$ patients. There was a distinct circadian periodicity, with the attacks occurring predictably during the night in 24 (92.3\%) patients. Regarding concomitant headaches, six (23.0\%) patients suffered from episodic migraine and five (19.2\%) patients from chronic migraine. Interestingly, one developed chronic migraine at the same time as $\mathrm{PTH}-\mathrm{CH}$, whereas three patients also suffered from post-traumatic headache with a chronic migraine phenotype but related to another head injury. All patients could clearly distinguish $\mathrm{CH}$ attacks from migraine pain. Of the 26 patients with PTH-CH, a family history of $\mathrm{CH}$ in at least one first-degree relative was confirmed in $15.3 \%$ of patients, consisting of 4 individuals from 3 families. Two patients were brother and sister and their mother also suffered from $\mathrm{CH}$, confirmed by us at the clinic. The affected relatives of the two remaining patients with $\mathrm{PTH}-\mathrm{CH}$ with probable family history had passed away and therefore were not available for interview. Nevertheless, one (the mother of the patient with PTH-CH) had previously been diagnosed with $\mathrm{CH}$ and the other (the uncle of the patient with PTH-CH) had a history that was highly suspicious for $\mathrm{CH}$ (extremely severe headaches localised behind the eye and occurring during the night).

Regarding the response to medical treatment, 2 (7.6\%) patients were considered as intractable to acute treatment and $11(42.3 \%)$ as intractable to preventive treatment. Three of them benefited from invasive neuromodulation, including occipital nerve stimulation, sphenopalatine ganglion stimulation and ventral tegmental region deep brain stimulation.

\section{Head trauma and its relationship to headache}

The details on head trauma are summarised in table 2 . The vast majority of patients $(n=23,88.4 \%)$ sustained mild injury. Three $(11.6 \%)$ traumas were considered as severe due to the duration of loss of consciousness and/or presence of anterograde amnesia. No intracranial haemorrhage was reported. There were however two skull fractures described in our cohort. The different mechanisms of head injury included road traffic accident $(n=5)$, mechanical fall $(n=4)$, collision with an object $(n=7)$, assault $(n=3)$ or direct penetration of the head by metal or glass $(n=2)$. In addition, dental extractions $(n=5)$ were assessed as the ICHD defines head injury as 'penetration of the head by a foreign body' but were not included for comparison with the control cohort, considering that they were not strictly identical to other patients with PTH-CH. Following the trauma, five patients had bruising and three had deep laceration. The first $\mathrm{CH}$ attacks occurred a few hours after the trauma in seven patients and immediately after it in five patients. The $\mathrm{CH}$ attacks were ipsilateral to the head injury in all patients in whom the trauma was clearly onesided, except one (patient 2). Due to a wider and often bilateral injury, laterality was indeterminate in 11 patients.

\section{Comparison with control cohort}

Univariate analyses comparing the 21 patients with $\mathrm{PTH}-\mathrm{CH}$ (without dental extractions) with the control cohort are presented in table 3. A family history of $\mathrm{CH}$ was identified in $50(9.0 \%)$ patients according to medical records and confirmed over the phone or in person in $33(5.9 \%)$ patients of the total control cohort. Regarding the headache diagnosis, patients with PTH-CH were more likely to be diagnosed with the $\mathrm{CCH}$ phenotype $(80.9 \%$ vs $50.4 \% ; \mathrm{p}=0.006)$ and with associated chronic migraine $(19.0 \%$ vs $7.6 \% ; \mathrm{p}=0.05)$ than the control cohort. Univariate analysis revealed statistical differences in terms of location, cranial autonomic features and response to preventive treatment. Indeed, a parietal location of referred pain, which was the most common site of injury, was more common in the PTH-CH group (38.0\% vs $16.0 \% ; \mathrm{p}=0.008)$. They were at a higher risk of being intractable to preventive treatment than the control cohort $(42.8 \%$ vs $16.0 \% ; \mathrm{p}=0.002)$. The results remained unchanged after including the five dental extractions cases (data not shown).

For multivariate analysis, we first approximately balanced the two classes with the ROSE algorithm and obtained a new sample generated from the first one with 270 patients with $\mathrm{PTH}-\mathrm{CH}$ and 304 patients with no PTH-CH. On this balanced data set, in a binary logistic framework, the LASSO selected 33 variables over the 37 original ones. Then we fed the 33 selected variables into a logistic regression. For the sake of simplicity, we only represented the items with significant results out of the 33 variables in figure 1 ( $\mathrm{n}=17$ items).

We found significant positive association between PTH-CH and family history of $\mathrm{CH}$ (OR $3.32 ; 95 \% \mathrm{CI} 1.31$ to 8.63 ), $\mathrm{CCH}$ phenotype (OR 3.29 ; 95\% CI 1.70 to 6.49 ), temporal location (OR 2.04; 95\% CI 1.10 to 3.84 ), parietal location (OR 14.82 ; $95 \%$ CI 6.32 to 37.39 ), eye oedema during attacks (OR 5.79; $95 \%$ CI 2.57 to 13.82 ), miosis during attacks (OR 11.24; 95\% CI 3.21 to 41.34 ), rhinorrhoea (OR 2.65; 95\% CI 1.26 to 5.86), facial sweating (OR 2.53; 95\% CI 1.33 to 4.93 ) and restlessness (OR 4.63; 95\% CI 1.16 to 22.19). Multivariate analysis confirmed intractability to acute (OR 12.34; 95\% CI 2.51 to 64.73 ) and preventive (OR 16.98; 95\% CI 6.88 to 45.52 ) treatment as independent characteristics of patients with PTH-CH. Associated chronic migraine had one of the highest ORs (10.35; 95\% CI 3.96 to 28.82) (table 4 and figure 1). Conversely, patients with $\mathrm{PTH}-\mathrm{CH}$ were less likely to present with frontal referred pain (OR 0.09 ; 95\% CI 0.03 to 0.19 ), absence of cranial autonomic features during attacks (OR 0.09 ; 95\% CI 0.01 to 0.60 ), conjunctival injection (OR 0.33 ; 95\% CI 0.12 to 0.86 ), lacrimation (OR $0.25 ; 95 \%$ CI 0.09 to 0.66 ) or flush (OR $0.28 ; 95 \% \mathrm{CI}$ 0.12 to 0.59$)$.

\section{DISCUSSION}

To our knowledge, this is the largest series of PTH-CH reported to date. Here, we describe the clinical characteristics of 26 patients with PTH-CH who developed headaches within 7 days of head trauma that strictly fulfil the ICHD-3 criteria for both $\mathrm{PTH}$ and $\mathrm{CH}$. We have kept five patients who underwent dental extractions apart, considering that these cases have a postsurgical rather than a typical post-traumatic aetiology. Univariate analysis comparing the remaining 21 patients with $\mathrm{PTH}-\mathrm{CH}$ with a control cohort of 553 patients with primary $\mathrm{CH}$ revealed 


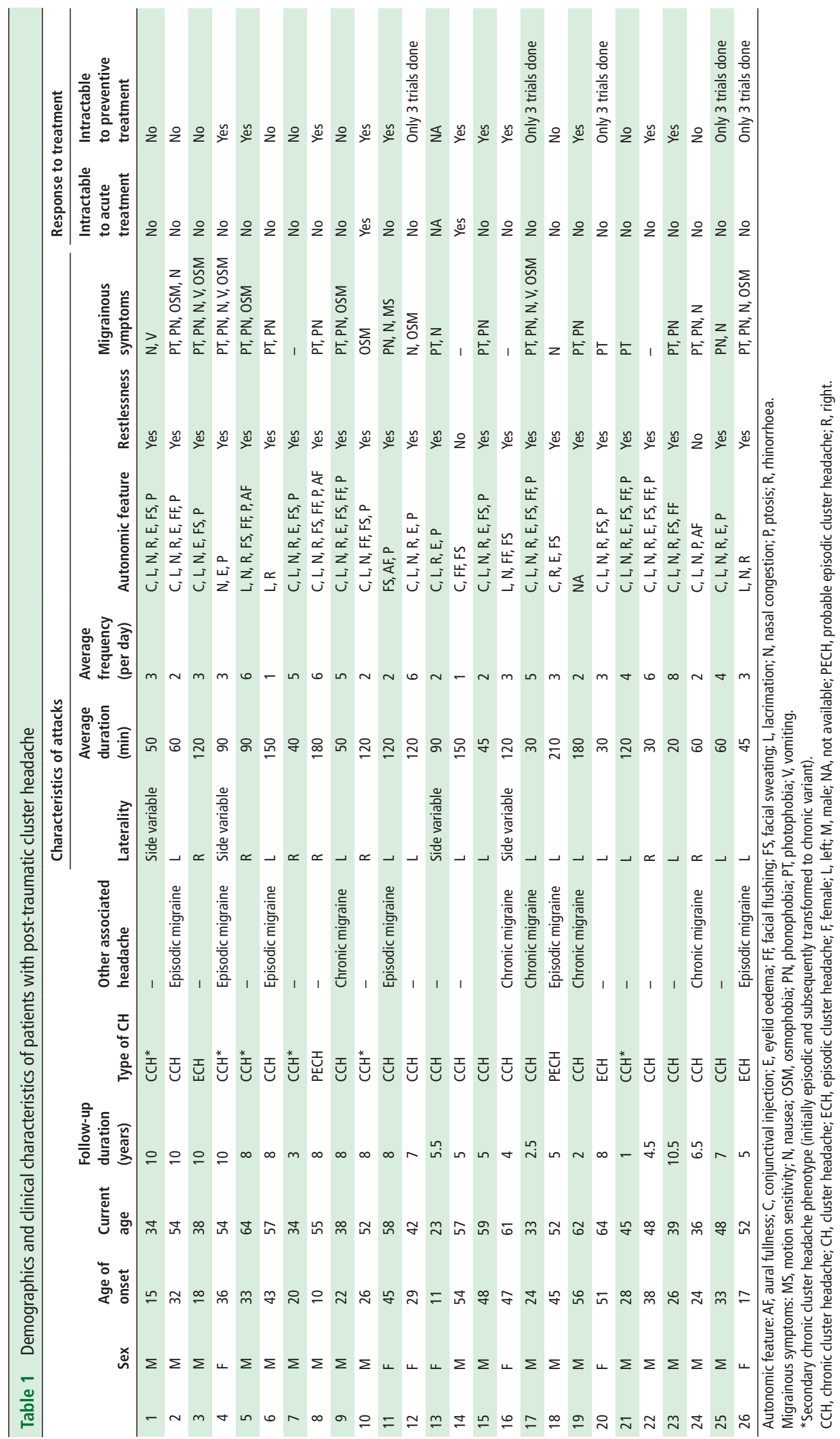




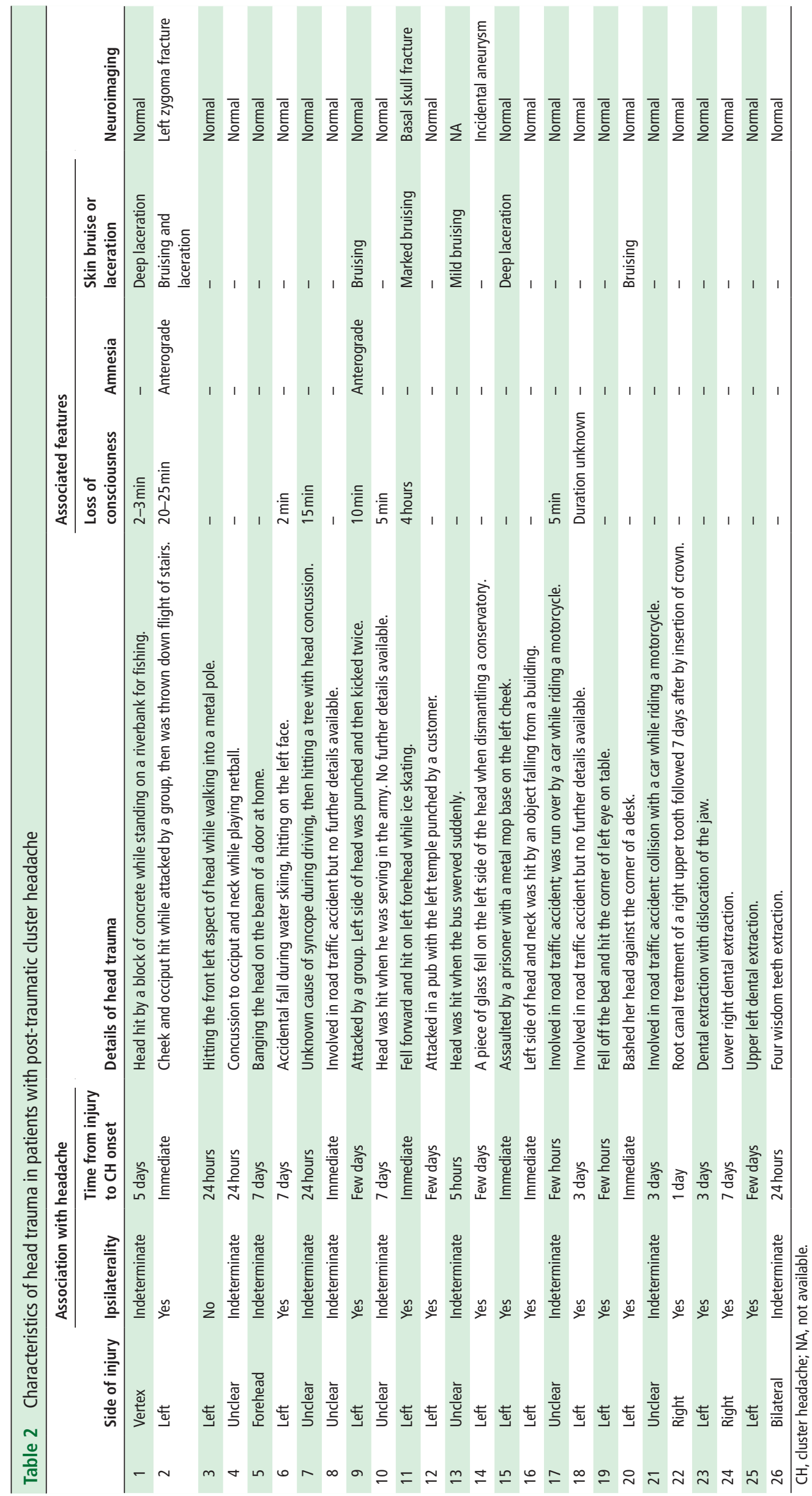


Table 3 Univariate analysis results of comparison between PTH-CH and primary $\mathrm{CH}$ (control cohort)

\begin{tabular}{|c|c|c|c|}
\hline & PTH-CH & Control cohort & \\
\hline & $(n=21)$ & $(n=553)$ & $P$ value \\
\hline Demographics & & & \\
\hline Age (years) & $49.3 \pm 11.9$ & $48.8 \pm 12.4$ & 0.56 \\
\hline Gender (male/female) & $15 / 6$ & $379 / 174$ & 0.77 \\
\hline Follow-up duration (years) & $6.47 \pm 3.0$ & $6.04 \pm 2.8$ & 0.43 \\
\hline Age at onset (years) & $32.9 \pm 14.4$ & $31.3 \pm 13.6$ & 0.56 \\
\hline Family history of $\mathrm{CH}$ & $4(19.0)$ & $50(9.0)$ & 0.12 \\
\hline Headache diagnosis & & & \\
\hline $\mathrm{CH}$ & & & \\
\hline $\mathrm{ECH}$ and/or PECH & $4(19.1)$ & $274(49.5)$ & 0.006 \\
\hline $\mathrm{CCH}$ and/or PCCH & $17(80.9)$ & $279(50.4)$ & \\
\hline Associated migraine & & & \\
\hline Episodic migraine & $5(23.8)$ & $99(17.9)$ & 0.49 \\
\hline Chronic migraine & $4(19.0)$ & $42(7.6)$ & 0.05 \\
\hline Duration and frequency of a & & & \\
\hline Usual duration (min) & $97.8 \pm 56.0$ & $90.8 \pm 64.0$ & 0.44 \\
\hline Average frequency per day & $3.0 \pm 1.5$ & $2.8 \pm 2.1$ & 0.08 \\
\hline Laterality & & & \\
\hline Strictly unilateral & $16(76.1)$ & $444(80.2)$ & 0.64 \\
\hline Side variable & $5(23.8)$ & $91(16.4)$ & 0.37 \\
\hline Bilateral & 0 & $18(3.2)$ & 0.4 \\
\hline Site and referred pain & & & \\
\hline Orbital/retro-orbital & 11 & 398 & 0.05 \\
\hline Frontal & 6 & 176 & 0.75 \\
\hline Temple & 11 & 267 & 0.71 \\
\hline Parietal & 8 & 89 & 0.008 \\
\hline Occiput & 5 & 111 & 0.67 \\
\hline Cranial autonomic features & tlessness & & \\
\hline Ptosis & 16 & 304 & 0.05 \\
\hline Eyelid oedema & 12 & 192 & 0.03 \\
\hline Conjunctival injection & 15 & 365 & 0.6 \\
\hline Miosis & 1 & 22 & 0.85 \\
\hline Lacrimation & 16 & 428 & 0.89 \\
\hline Nasal blockage & 14 & 320 & 0.42 \\
\hline Rhinorrhoea & 13 & 330 & 0.83 \\
\hline Facial sweating & 14 & 265 & 0.09 \\
\hline Facial flush & 9 & 208 & 0.63 \\
\hline Aural fullness & 3 & 86 & 0.87 \\
\hline Restlessness & $20(95.2)$ & $428(83.9)$ & 0.06 \\
\hline Response to medical treatm & & & \\
\hline Acute treatment & & & \\
\hline Intractable & 2 & 16 & 0.23 \\
\hline Indeterminate & 4 & 130 & \\
\hline Responsive & 16 & 407 & \\
\hline Preventive treatment & & & \\
\hline Intractable & 9 & 89 & 0.002 \\
\hline Indeterminate & 8 & 210 & \\
\hline Responsive & 4 & 254 & \\
\hline
\end{tabular}

Mean \pm SD for quantitative data; $\mathrm{n}(\%)$ for qualitative data.

$\mathrm{CCH}$, chronic cluster headache; $\mathrm{CH}$, cluster headache; $\mathrm{ECH}$, episodic cluster headache; $\mathrm{PCCH}$, probable chronic cluster headache; $\mathrm{PECH}$, probable episodic cluster headache; PTH-CH, post-traumatic headache with cluster headache phenotype.

that patients with $\mathrm{PTH}-\mathrm{CH}$ were more likely to have the chronic variant of $\mathrm{CH}$, parietal site of pain, prominent cranial autonomic features particularly ptosis and eyelid oedema, intractability to

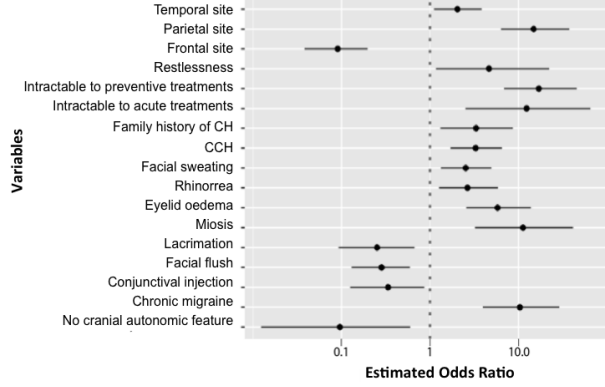

Figure 1 Estimated OR in log-10 scale of each selected item entered into the logistic regression model with significant result $(n=17)$. Eventually, we used the built-in ROSE.eval function in order to estimate our model accuracy. The ROSE.eval function implemented a ROSE version of holdout: the logistic regression was fitted on one sample generated by ROSE and tested on the original data set. The threshold for the logistic regression was set to 0.5 . It turned out that the area under the curve was 0.874 , which shows the accuracy of the estimated model. $\mathrm{CCH}$, chronic cluster headache; $\mathrm{CH}$, cluster headache.

preventive treatments, and have chronic migraine. A multivariate logistic regression model comparing the 21 patients with PTH-CH with the 553 patients with primary $\mathrm{CH}$ confirmed that patients with PTH-CH were more likely to have a family history of $\mathrm{CH}$, the chronic variant of $\mathrm{CH}$, temporal and parietal site of pain, prominent cranial autonomic features (particularly eyelid oedema, miosis, rhinorrhoea and facial sweating), restlessness, and intractability to acute and preventive treatments, as well as associated chronic migraine. These findings suggest that $\mathrm{PTH}-\mathrm{CH}$ has a distinct clinical phenotype with more severe $\mathrm{CH}$ phenotype that is less responsive to treatments compared with primary $\mathrm{CH}$. Furthermore, it implies that $\mathrm{PTH}-\mathrm{CH}$ may have an alternative pathophysiological mechanism with its own evolutive profile.

In primary $\mathrm{CH}$, wide activation of ipsilateral trigeminal nociceptive pathways, involving the trigeminal-autonomic reflex, leads to central activation through the trigeminal nucleus caudalis and the superior salivatory nucleus in the brainstem. ${ }^{821}$ In contrast, it is suggested that PTH results from more localised changes in the trigeminal pathway due to direct damage at the site of trauma. ${ }^{22}{ }^{23}$ Factors including axonal injury, reduced cerebral circulation and the inappropriate release of local

Table 4 Results of multivariate logistic regression model comparing post-traumatic headache with cluster headache phenotype $(n=21)$ and primary cluster headache (control cohort; $n=553$ )

\begin{tabular}{lrlr}
\hline Predictive factor & OR & 95\% Cl & P value \\
\hline Family history of cluster headache & 3.32 & 1.31 to 8.63 & 0.012 \\
\hline Chronic cluster headache phenotype & 3.29 & 1.70 to 6.49 & $<0.001$ \\
Temporal location & 2.04 & 1.10 to 3.84 & 0.024 \\
Parietal location & 14.82 & 6.32 to 37.39 & $<0.001$ \\
Presence of eye oedema & 5.79 & 2.57 to 13.82 & $<0.001$ \\
Presence of miosis & 11.24 & 3.21 to 41.34 & $<0.001$ \\
Presence of rhinorrhoea & 2.65 & 1.26 to 5.86 & 0.013 \\
Presence of facial sweating & 2.53 & 1.33 to 4.93 & 0.005 \\
Restlessness & 4.63 & 1.16 to 22.19 & 0.039 \\
Associated chronic migraine & 10.35 & 3.96 to 28.82 & $<0.001$ \\
Intractable to acute treatment & 12.34 & 2.51 to 64.73 & 0.002 \\
Intractable to preventive treatment & 16.98 & 6.88 to 45.52 & $<0.001$ \\
\hline
\end{tabular}


neurotransmitters are likely to play a role in the initial emergence of PTH-CH. ${ }^{4}{ }^{8}$ Consistent with previous reports, there was a propensity for referred pain in the parietal and temporal regions during PTH-CH attacks in our cohort. ${ }^{24}$ This likely reflects localised changes. Furthermore, the majority of patients with strictly unilateral headache reported pain on the side ipsilateral to the injury. Although the pain distribution may lead to attribution to some event in the past, this is consistent with previous findings and supports the hypothesis of peripheral involvement in $\mathrm{PTH}-\mathrm{CH}$ pathogenesis via direct damage to local nociceptive structures. ${ }^{25} 26$

Nevertheless, diffuse changes such as excessive neuronal depolarisation and the release of excitotoxic neurotransmitters may underpin the pathogenesis of PTH-CH. ${ }^{4}$ The sensitisation of central trigeminal neurons in PTH as a consequence of the initial trauma-related inflammatory process within the cranial meninges and the calvarial periosteum may also be involved. ${ }^{27}{ }^{28}$ During primary $\mathrm{CH}$ attacks, the ipsilateral cranial autonomic features testify of cranial parasympathetic activation and sympathetic hypofunction, due to a central disinhibition of the trigeminal autonomic reflex. ${ }^{29}$ The more frequent eyelid oedema, miosis, rhinorrhoea and facial sweating in PTH-CH compared with primary $\mathrm{CH}$ in our study may reveal a marked disinhibition of pain pathways after trauma. Such long-term modulation of central pain pathways in $\mathrm{CH}$ following trauma would also explain the vast majority of chronic variant found in our study. Indeed, more than $80 \%$ of patients with PTH-CH suffered from the chronic variant and 15 of them developed the chronic form at onset, without any intermittent pain-free period. This further supports a continuous sensitivity to common headache triggering factors. ${ }^{28} \mathrm{PTH}$ with a chronic migraine phenotype co-occurred in 19\% (4 of 21) of our cohort, exceeding the estimated 3\% incidence in the general population and reaching the high OR of 10.35 in multivariate analysis. ${ }^{30}$ Although PTH can be heterogenous, patients could clearly distinguish their $\mathrm{CH}$ attacks from their migraine pain, arguing more for true concomitant headaches than one heterogeneous phenotype of the same type of headache. Similarly, this may result from central sensitisation with dysfunction of brainstem antinociceptive centres (implicated in migraine) as well as the hypothalamus (implicated in $\mathrm{CH}$ ). ${ }^{31}$ Thus, head trauma may lead to global alteration in the 'pain neuromatrix', with concomitant forms of $\mathrm{CH}$ and migraine. The marked intractability of PTH-CH to treatment, both acute and preventive, could be another direct consequence of these modifications.

The rarity of $\mathrm{CH}$ following trauma, compared with migraine and tension-type headache, remains unexplained. ${ }^{8}$ Nevertheless, in a study in military service members with a history of mild traumatic brain injury $(\mathrm{n}=95)$, TAC type $(\mathrm{n}=6)$ was the second most prevalent, after migraine type. ${ }^{10}$ The emergence of $\mathrm{CH}$ phenotype may be due to a genetic susceptibility. We found that four (15.3\%) patients with PTH-CH had family history of $\mathrm{CH}$ in first-degree relatives, exceeding the estimated prevalence of $\mathrm{CH}$ of $1.2 \%$ in the general population. Interestingly, there were three times the odds of having a family history of $\mathrm{CH}$ in case of PTH-CH. This implies that genetic risk in combination with an environmental trigger, such as head trauma, may be required to develop the phenotype.

PTH-CH may occur only in cases where there is hypothalamic disruption secondary to trauma and subsequent reorganisation. ${ }^{32}$ Indeed, the somatosensory system is capable of reorganisation following peripheral denervation and pain. ${ }^{33} 34$ There is supporting evidence for the involvement of posterior hypothalamus in primary $\mathrm{CH}$ attacks. ${ }^{29}{ }^{35} \mathrm{Head}$ trauma can lead to a loss of hypocretin-producing neurons in the posterolateral hypothalamus, and $\mathrm{CH}$ attacks are known to occur when the orexinergic system is downregulated. ${ }^{36}{ }^{37}$ Indeed, the circadian periodicity is one of the hallmarks of $\mathrm{CH}$ attacks and concerns $70 \%$ of patients. ${ }^{38}$ The unusual high rate of circadian periodicity, documented in $92 \%$ of our patients with $\mathrm{PTH}-\mathrm{CH}$, may be suggestive of hypothalamic dysfunction related to trauma.

Weaknesses of this study include that our data were collected at a tertiary headache centre. Comparison with a large control cohort, who attended the same clinic during the same period, minimises this referral bias. Retrospective data may involve memory biases, but the rarity of the PTH-CH entity makes a prospective study unlikely to be feasible. Disability from PTH is compounded by coexisting post-traumatic stress disorder. ${ }^{39}$ Unfortunately, we did not formally perform psychological assessment or comparison with primary $\mathrm{CH}$ in terms of quality of life. Post-traumatic sleep and mood disturbances can plausibly influence the development and perpetuation of headache, but also the response to treatment. ${ }^{15}$ In terms of intractability, a large number of patients were classified as indeterminate due to incomplete trials. Finally, rather broad CIs were found after logistic regression owing to a small number of patients with $\mathrm{PTH}-\mathrm{CH}$. In order to take into account those imbalanced groups and give relevant results, we used several powerful statistical strategies such as ROSE and LASSO algorithms.

The strengths of the study include the large sample of patients who strictly fulfil the ICHD criteria for both PTH and $\mathrm{CH}$, allowing us to consider our conclusions as strongly reliable. An association between head injury and $\mathrm{CH}$ has already been described in the past, but most injuries were quite remote from the first $\mathrm{CH}$ attack. ${ }^{8}$ The definition of PTH implies a close temporal relationship, established as the occurrence of headache within 7 days after head trauma. This stipulation might be somewhat arbitrary but yields a stronger evidence of causation, leading to a higher specificity of the ICHD criteria. ${ }^{1}$ Indeed, a high proportion of patients with $\mathrm{CH}$ sustained head injury several years prior to $\mathrm{CH}$ onset, suggesting an association between trauma and $\mathrm{CH}$, which goes beyond the rare occurrence of PTH-CH cases, thus raising the hypothesis of distinctive lifestyles in patients with $\mathrm{CH}^{40}$

In conclusion, this series is the first to describe in detail the specific clinical characteristics of PTH-CH. We demonstrated that PTH-CH is more likely to present as chronic form, with marked cranial autonomic features and temporoparietal location of attacks in patients with family history of $\mathrm{CH}$. They have a considerably higher risk of intractability to treatment and associated chronic migraine. This unique evolutive profile possibly reflects sensitisation of the pain neuromatrix and hypothalamus following trauma.

Acknowledgements We would like to thank our headache specialist nurses for their help with completion of the clinical database and management of patients. We also thank the patients and their families for their help with this project.

Contributors LG: collection, analysis and interpretation of data, and drafting and revision of the manuscript. EO: analysis and interpretation of data, and drafting and revision of the manuscript. C-KC: collection and analysis of data. LA and TMPN: statistical analysis and interpretation of data, and drafting of the manuscript. MSM: study concept, recruitment of subjects, interpretation of data and manuscript revision.

Funding The authors have not declared a specific grant for this research from any funding agency in the public, commercial or not-for-profit sectors.

Competing interests MSM serves on the advisory board for Allergan, St Jude Medical, Medtronic and Eli Lilly, and has received payment for the development of educational presentations from Allergan, St Jude Medical, Medtronic and electroCore.

Patient consent for publication Not required.

Ethics approval Ethics board approval was obtained from the National Hospital for Neurology and Neurosurgery Research Ethics Committee, London, UK (REC number: 07/Q0512/26). 
Provenance and peer review Not commissioned; externally peer reviewed.

Data availability statement Data are available upon reasonable request. De-identified database and statistical analysis plan will be shared upon reasonable request for 2 years after publication.

Open access This is an open access article distributed in accordance with the Creative Commons Attribution Non Commercial (CC BY-NC 4.0) license, which permits others to distribute, remix, adapt, build upon this work non-commercially, and license their derivative works on different terms, provided the original work is properly cited, appropriate credit is given, any changes made indicated, and the use is non-commercial. See: http://creativecommons.org/licenses/by-nc/4.0/.

\section{ORCID iD}

Manjit Singh Matharu http://orcid.org/0000-0002-4960-2294

\section{REFERENCES}

1 Headache classification Committee of the International headache Society (IHS) the International classification of headache disorders, 3rd edition. Cephalalgia 2018;38:1-211.

2 Sheftell FD, Tepper SJ, Lay CL, et al. Post-Traumatic headache: emphasis on chronic types following mild closed head injury. Neuro/ Sci 2007;28:S203-7.

3 Nampiaparampil DE. Prevalence of chronic pain after traumatic brain injury: a systematic review. JAMA 2008;300:711-9.

4 Packard RC, Ham LP. Pathogenesis of posttraumatic headache and migraine: a common headache pathway? Headache 1997;37:142-52.

5 Matharu MJ, Goadsby PJ. Post-Traumatic chronic paroxysmal hemicrania (CPH) with aura. Neurology 2001;56:273-5.

6 Lambru G, Castellini P, Manzoni GC, et al. Post-Traumatic cluster headache: from the periphery to the central nervous system? Headache 2009;49:1059-61.

7 Putzki N, Nirkko A, Diener HC. Trigeminal autonomic cephalalgias: a case of posttraumatic SUNCT syndrome? Cephalalgia 2005:25:395-7.

8 Lambru G, Matharu M. Traumatic head injury in cluster headache: cause or effect? Curr Pain Headache Rep 2012;16:162-9.

9 Manzoni GC, Lambru G, Torelli P. Head trauma and cluster headache. Curr Pain Headache Rep 2006;10:130-6.

10 Finkel AG, Yerry JA, Klaric JS, et al. Headache in military service members with a history of mild traumatic brain injury: a cohort study of diagnosis and classification. Cephalalgia 2017;37:548-59.

11 Matharu M, Lambru G. Trigeminal autonomic cephalalgias: a review of recent diagnostic, therapeutic and pathophysiological developments. Ann Indian Acad Neurol 2012;15:51

12 Yang F-C, Chou K-H, Fuh J-L, et al. Altered hypothalamic functional connectivity in cluster headache: a longitudinal resting-state functional MRI study. J Neurol Neurosurg Psychiatry 2015;86:437-45.

13 Kjeldgaard D, Forchhammer H, Teasdale T, et al. Chronic post-traumatic headache after mild head injury: a descriptive study. Cephalalgia 2014;34:191-200.

14 Yilmaz T, Roks G, de Koning M, et al. Risk factors and outcomes associated with posttraumatic headache after mild traumatic brain injury. Emerg Med J EMJ.

15 Minen MT, Boubour A, Walia $\mathrm{H}$, et al. Post-Concussive syndrome: a focus on posttraumatic headache and related cognitive, psychiatric, and sleep issues. Curr Neurol Neurosci Rep 2016:16:100

16 Mitsikostas DD, Edvinsson L, Jensen RH, et al. Refractory chronic cluster headache: a consensus statement on clinical definition from the European headache Federation. $J$ Headache Pain 2014;15:79.
17 Goadsby PJ, Schoenen J, Ferrari MD, et al. Towards a definition of intractable headache for use in clinical practice and trials. Cephalalgia 2006;26:1168-70.

18 Schürks M, Kurth T, de Jesus J, et al. Cluster headache: clinical presentation, lifestyle features, and medical treatment. Headache 2006;46:1246-54.

19 Lunardon N, Menardi G, Torelli N. Rose: a package for binary imbalanced learning. $R J$ 2014;6:79.

20 Tibshirani R. Regression shrinkage and selection via the LASSO. J R Stat Soc Ser B 1996;58:267-88

21 May A, Goadsby PJ. The trigeminovascular system in humans: pathophysiologic implications for primary headache syndromes of the neural influences on the cerebral circulation. J Cereb Blood Flow Metab 1999;19:115-27.

22 Turkewitz LJ, Wirth O, Dawson GA, et al. Cluster headache following head injury: a case report and review of the literature. Headache 1992;32:504-6.

23 Benromano T, Defrin R, Ahn AH, et al. Mild closed head injury promotes a selective trigeminal hypernociception: implications for the acute emergence of post-traumatic headache: closed head injury promotes trigeminal hypernociception. Eur J Pain 2015:19:621-8.

24 Defrin R. Chronic post-traumatic headache: clinical findings and possible mechanisms J Man Manip Ther 2014:22:36-43.

25 Rogado AZ, Graham JR. Through a glass darkly. Headache 1979;19:58-62.

26 Lance JW, Anthony M. Migrainous neuralgia or cluster headache? J Neuro/ Sci 1971;13:401-14

27 Burstein R. Deconstructing migraine headache into peripheral and central sensitization. Pain 2001;89:107-10.

28 Bree D, Levy D. Development of CGRP-dependent pain and headache related behaviours in a rat model of concussion: implications for mechanisms of posttraumatic headache. Cephalalgia 2018;38:246-58.

29 Leone M, Bussone G. Pathophysiology of trigeminal autonomic cephalalgias. Lancet Neurol 2009;8:755-64.

30 May A, Schulte LH. Chronic migraine: risk factors, mechanisms and treatment. Nat Rev Neurol 2016;12:455-64.

31 May A, Goadsby PJ. Hypothalamic involvement and activation in cluster headache. Curr Pain Headache Rep 2001;5:60-6.

32 Tanriverdi F, Unluhizarci K, Kelestimur F. Pituitary function in subjects with mild traumatic brain injury: a review of literature and proposal of a screening strategy. Pituitary 2010;13:146-53.

33 Saab CY. Pain-Related changes in the brain: diagnostic and therapeutic potentials. Trends Neurosci 2012;35:629-37.

34 Sörös P, Knecht S, Bantel C, et al. Functional reorganization of the human primary somatosensory cortex after acute pain demonstrated by magnetoencephalography. Neurosci Lett 2001:298:195-8.

35 May A, Bahra A, Büchel C, et al. Pet and MRA findings in cluster headache and MRA in experimental pain. Neurology 2000;55:1328-35.

36 Baumann CR, Bassetti CL, Valko PO, et al. Loss of hypocretin (orexin) neurons with traumatic brain injury. Ann Neurol 2009:66:555-9.

37 Holland PR, Goadsby PJ. Cluster headache, hypothalamus, and orexin. Curr Pain Headache Rep 2009:13:147-54.

38 Almoznino G, Benoliel R, Sharav Y, et al. Sleep disorders and chronic craniofacial pain: characteristics and management possibilities. Sleep Med Rev 2017;33:39-50.

39 Roper LS, Nightingale P, Su Z, et al. Disability from posttraumatic headache is compounded by coexisting posttraumatic stress disorder. J Pain Res 2017;10:1991-6.

40 Lambru G, Castellini P, Manzoni GC, et al. Mode of occurrence of traumatic head injuries in male patients with cluster headache or migraine: is there a connection with lifestyle? Cephalalgia 2010;30:1502-8. 